\title{
Anesthetic Management of a Case of Hemophagocytic Lymphohistiocytosis
}

\author{
Gentle S Shrestha ${ }^{1}$, Harmeet Gulati ${ }^{2}$, Binita Acharya ${ }^{1}$, Bishesh S Poudyal ${ }^{3}$ \\ ${ }^{1}$ Department of Anesthesiology, Maharajgunj Medical Campus, Tribhuvan University Teaching Hospital, Institute \\ Of Medicine, Maharajgunj, Kathmandu, Nepal, ${ }^{2}$ Vardhman Mahavir Medical College \& Safdarjung Hospital, New \\ Delhi, India, ${ }^{3}$ Civil Service Hospital, Kathmandu, Nepal
}

\section{Corresponding author:}

Gentle S Shrestha, MD, FACC

Department of Anesthesiology, Maharajgunj Medical Campus, Tribhuvan University Teaching Hospital, Institute of Medicine, Maharajgunj, Kathmandu, Nepal

Email: gentlesunder@hotmail.com

Submitted : Feb 3, 2020

Accepted : Mar 28, 2020

\begin{abstract}
Hemophagocytic Lymphohistiocytosis $(\mathrm{HLH})$ is characterized by an overwhelming activation of histiocytes and T lymphocytes leading to fulminant hemophagocytosis and organ damage. Anesthetic management of patients with HLH is associated with challenges such as pancytopenia, hemodynamic instability and infections. In addition to sepsis, metabolic acidosis, disseminated intravascular coagulation, multi-organ involvement with an exacerbation of symptoms with the use of anesthetic agents poses significant risk perioperatively. We present a case planned for excision of pilonidal sinus. The surgery was performed after thorough workup and with the patient in remission. Elective procedures should ideally be performed with patients in remission. Cases receiving concurrent therapy with immunomodulators and steroids require special attention due to possible interaction with anesthetic agents and the need for perioperative steroid supplementation.
\end{abstract}

Keywords: Anesthetic management, hemophagocytic lymphohystiocytosis, pancytopenia, steroids

\section{INTRODUCTION}

$\mathrm{H}$ emophagocytic lymphohistiocytosis (HLH) represents an uncontrolled inflammatory condition characterized by fever, cytopenia, hepatosplenomegaly and phagocytosis by activated macrophages, facilitated by impaired function of natural killer cells and cytotoxic $T$ cell lymphocytes. $\mathrm{HLH}$ has a broad clinical phenotype overlapping with systemic inflammatory response syndrome, sepsis, and multiorgan dysfunction syndrome. Classically, although more commonly seen in young children, it is increasingly being diagnosed in adults nowadays.

$\mathrm{HLH}$ is classified as primary when attributable to an underlying genetic abnormality and secondary when precipitated by infection, autoimmune, rheumatologic, malignant, or metabolic conditions. ${ }^{1}$

The diagnosis of $\mathrm{HLH}$ is based on fulfilling one or both of the criteria established by the Histiocyte Society (Table 1). ${ }^{2}$

Specific treatment modalities include dexamethasone or methylprednisolone as steroids, and immunomodulators like cyclosporine, etoposide, infliximab, and rituximab. ${ }^{3}$ 
Table 1. Diagnostic guidelines for hemophagocytic lymphohistiocytosis

CRITERIA 1

Molecular diagnosis consistent with HLH

CRITERIA 2 (Five of the following criteria)

- Fever of $38.5^{\circ} \mathrm{C}$ or more

- Splenomegaly

- Cytopenias (affecting at least two of three cell lineages in the peripheral blood)

- Hemoglobin $<5.59 \mathrm{mmol} / \mathrm{L}$ (In infants <4 weeks, $\mathrm{Hb}<6.21 \mathrm{mmol} / \mathrm{L})$

- Platelets $<100$ cells per $10 \% / \mathrm{L}$

- Neutrophils $<1$ cell per 10\% $/ \mathrm{L}$

- Hypertriglyceridemia (fasting $>3 \mathrm{mmol} / \mathrm{L}$ )

- Hypofibrinogenemia (<1.7 mmol/L)

- Hemophagocytosis in bone marrow, spleen, lymph nodes, or liver

- Low or absent natural killer-cell activity

- Ferritin greater than $1123.5 \mathrm{pmol} / \mathrm{L}$

- Increased soluble CD25 concentration (alpha chain of soluble interleukin 2 receptor)

\section{CASE PRESENTATION}

A 19 years old male was planned for pilonidal sinus excision and flap surgery for a discharging anus sinus. He was diagnosed with HLH the previous year and was treated according to the HLH 2004 protocol with dexamethasone, etoposide and cyclosporine, the treatment having concluded 6 months back.

Preoperative investigations revealed a hemoglobin of $14.7 \mathrm{mg} / \mathrm{dl}$, total leucocyte count of 5600/ dl, 235000/dl platelets, prothrombin time of 11 seconds with an INR of 0.9, a serum biochemistry, ferritin, and fibrinogen within normal limits along with a normal chest X-ray and ECG findings.

Injection Hydrocortisone $100 \mathrm{mg}$ IV was administered during the surgery for perioperative stress. Subarachnoid block was performed using a 25G Quincke's needle via midline approach with 2 $\mathrm{ml}$ of $0.5 \%$ hyperbaric Bupivacaine. Sensory block was tested to T10 level and grade 4 motor block was achieved. The surgery was uneventful.

The patient was observed for 3 hours in postanesthesia care unit and subsequently shifted to the ward. Patient was discharged home on the third postoperative day.

\section{DISCUSSION}

Anesthetic concerns for a case of HLH include the presence of coexisting infection, an inflammatory disease process, and malignancy, and entails a high risk of perioperative flare up. Concurrently, the patient may be in sepsis/septic shock. HLH is frequently associated with coagulopathy with a deranged prothrombin time and high chances of the patient deteriorating to $\mathrm{DIC} .{ }^{4}$

$\mathrm{HLH}$ is also associated with deranged electrolytes, triglycerides, and liver function tests. Pulmonary involvement is frequent $(42 \%)$, and symptoms can include cough, dyspnea, and respiratory failure thus necessitating the need for possible mechanical ventilation postoperatively. ${ }^{5}$

CNS involvement (25\%) is heterogeneous and includes coma, seizures, meningitis, encephalomyelitis, cavernous sinus syndrome, or cerebral hemorrhage. Renal involvement may present as acute renal failure or nephrotic syndrome. Cardiovascular involvement includes features of cardiac failure and pericarditis.

Anesthetic management of a case with HLH is complex and various factors must be taken into account before making decisions. It is essential to determine whether the case is in active phase or remission phase. ${ }^{2}$

Elective procedures should be scheduled during remission phase of disease. Anesthetic management for a patient in remission should primarily be focussed on prevention of relapse. Preoperatively, a thorough respiratory examination should be done and chest radiograph obtained to estimate the extent of pulmonary involvement. In case of extensive involvement, patient may need post-operative mechanical ventilatory support. Sedative drugs (benzodiazepines) can worsen respiratory insufficiency and should be avoided in these patients.

Perioperative antibiotics and steroid coverage must be adequate so as to mitigate the risk of a flare up. Body temperature should be monitored to avoid hyperthermia and associated increased oxygen demand. Various trigger factors such as nonsteroidal anti-inflammatory agents, drugs releasing histamine (Morphine, Atracurium) and hypothermia need to be avoided in the perioperative period.

Patients in active phase of the disease may be encountered in an emergent setting and the anesthetic management of such a case needs to be aggressive. The active phase of HLH may mimic febrile illness, liver diseases, multiple organ dysfunction syndrome, encephalitis, and sepsis. One needs to have a high degree of suspicion in order to correctly diagnose HLH in such scenarios. Laboratory investigations such as serum ferritin, fasting triglycerides, soluble CD25 and fibrinogen can differentiate HLH from other conditions.6 Additionally, a complete blood count and a bone marrow aspirate, should be done in order to reach a definitive diagnosis. Prompt initiation of immunochemotherapy is essential for survival.

Active phase of $\mathrm{HLH}$ is often associated with a low 
platelet count and coagulation dysfunction due to liver failure. ${ }^{7}$ Regional anesthesia should be avoided in these patients. Assuring availability of fresh frozen plasma, platelet and concentrated red cells, tranexamic acid and coagulation factors may be necessary.

Due to life threatening nature of the active disease, arterial cannulation for invasive blood pressure measurement and central venous line placement is recommended. In case of cardiac failure, intraoperative transesophageal echocardiography can be useful. Nitrous oxide is better avoided in patients with cardiac failure and/or pericardial effusion, because of cardio-depressant effects. Additionally, patients may require a lower dose of propofol and opiates.

During relapse many of these patients have clinical and radiologic findings similar to the acute respiratory distress syndrome, with alveolar infiltrates and pleural effusions.8,9 Ultrasonography may show gall bladder thickening, hyperechoic kidneys, ascites, and hepatosplenomegaly. Case series of adults treated with a variety of regimens report a 30-day mortality of $20 \%$ to $44 \%$ and overall mortality of $50 \%$ to $75 \% .{ }^{10}$ Anesthetic management of a case in relapse should be managed as aggressively as an active case as these patients have a higher overall risk of mortality.

\section{CONCLUSION}

Patients with HLH pose unique challenges for anesthesiologists. HLH can be associated with myriad of problems like coagulopathy, dyselectrolytemia, hepatic dysfunction, pulmonary and cardiovascular involvement, etc. When possible, elective surgeries need to be planned during remission.

\section{CONFLICT OF INTEREST}

None declared.

\section{REFERENCES}

1. Ramos-Casals $M$, Brito-Zerón P, López-Guillermo A, Khamashta MA, Bosch X. Adult hemophagocytic syndrome. Lancet 2014;383:1503-16.

2. Chien CT, Lee FJ, Luk HN, Wu CC. Anesthetic management for cesarean delivery in a parturient with exacerbated hemophagocytic syndrome. Int J Obstet Anesth 2009;18:413-6.

3. George MR. Hemophagocytic lymphohistiocytosis: review of etiologies and management. J Blood Med 2014;5:69-86.

4. Jordan MB, Allen CE, Weitzman S, Filipovich $\mathrm{AH}, \mathrm{McClain} \mathrm{KL}$. How I treat hemophagocytic lymphohistiocytosis. Blood 2011;118:4041-52.

5. Fisman DN. Hemophagocytic syndromes and infection. Emerg Infect Dis 2000;6:601-8.

6. Emmenegger $U$, Schaer DJ, Larroche C, Neftel KA. Hemophagocytic syndromes in adults: current concepts and challenges ahead. Swiss Med Wkly 2005;135:299-314.

7. Imashuku S. Differential diagnosis of hemophagocytic syndrome: underlying disorders and selection of the most effective treatment. Int J Hematol 1997;66:135-51.

8. Tang YM, Xu XJ. Advances in hemophagocytic lymphohistiocytosis: pathogenesis, early diagnosis/differential diagnosis and treatment. Scientific World Journal 2011;11:697-708.

9. Henter JI, Horne A, Aricó M, Egeler RM, Filipovich $\mathrm{AH}$, Imashuku S, et al. HLH-2004: Diagnostic and therapeutic guidelines for hemophagocytic lymphohistiocytosis. Pediatr Blood Cancer 2007;48:124-31.

10. Schram AM, Berliner N. How I treathemophagocytic lymphohistiocytosis in the adult patient. Blood. 2015;125:2908-14 